\title{
Numerical solutions of higher order boundary value problems via wavelet approach
}

\author{
Shams UI Arifeen', Sirajul Haq ${ }^{1}$, Abdul Ghafoor ${ }^{2}$, Asad Ullah ${ }^{3}$, Poom Kumam, ${ }^{4,5} 6^{*}$ and Parin Chaipanya ${ }^{5,7}$
}

\section{"Correspondence:}

poom.kumam@kmutt.ac.th

${ }^{4}$ Fixed Point Theory and

Applications Research Group,

Center of Excellence in Theoretical

and Computational Science

(TaCS-CoE), Faculty of Science, King Mongkuts University of Technology Thonburi (KMUTT), 126 Pracha Uhit Rd, Bang Mod, Thung Khru, Bagnkok, 10140, Thailand

${ }^{5}$ Center of Excellence in Theoretical and Computational Science

(TaCS-COE), Faculty of Science, King Mongkuts University of Technology Thonburi (KMUTT), 126 Pracha Uhit Rd, Bang Mod, Thung Khru, Bagnkok, 10140, Thailand Full list of author information is available at the end of the article

\begin{abstract}
This paper presents a numerical scheme based on Haar wavelet for the solutions of higher order linear and nonlinear boundary value problems. In nonlinear cases, quasilinearization has been applied to deal with nonlinearity. Then, through collocation approach computing solutions of boundary value problems reduces to solve a system of linear equations which are computationally easy. The performance of the proposed technique is portrayed on some linear and nonlinear test problems including tenth, twelfth, and thirteen orders. Further convergence of the proposed method is investigated via asymptotic expansion. Moreover, computed results have been matched with the existing results, which shows that our results are comparably better. It is observed from convergence theoretically and verified computationally that by increasing the resolution level the accuracy also increases.
\end{abstract}

Keywords: Haar wavelet; Higher order boundary value problem; Quasilinearization

\section{Introduction}

Higher-order boundary value problems (HOBVPs) have widespread applications in diverse areas of science and engineering. Mostly, these problems arise in astronomy, fluid dynamics, astrophysics, hydrodynamics, beam theory, induction motors [1], and other branches of engineering sciences [2]. If an infinite smooth sheet of fluid is heated from below in the presence of a magnetic field in gravity direction, instability occurs. When this instability is ordinary convection, it is modeled through tenth order boundary value problems (BVPs). If instability sets are as over stability, it is modeled by twelfth order boundary value problem [3].

In generic cases, analytical solution of HOBVPs is a painstaking process, therefore researchers have turned their attention towards approximate solutions. For example, Syed et al. [4] applied modified variational iteration method (MVIM) for the solution of ninth and tenth order BVPs. Siddiqi et al. [5] solved eleventh order BVPs using variational iteration method. Mirmoradi et al. [6] implemented homotopy perturbation method (HPM) for twelfth order BVPs. Noor et al. [7] solved twelfth order BVPs via variational iteration method (VIM). Samaher [8] and his co-authors presented the solution of thirteenth order BVPs by modified Adomian decomposition method (MADM). Akram et al. $[9,10]$ applied

(c) The Author(s) 2021. This article is licensed under a Creative Commons Attribution 4.0 International License, which permits use sharing, adaptation, distribution and reproduction in any medium or format, as long as you give appropriate credit to the original author(s) and the source, provide a link to the Creative Commons licence, and indicate if changes were made. The images or other third party material in this article are included in the article's Creative Commons licence, unless indicated otherwise in a credit line to the material. If material is not included in the article's Creative Commons licence and your intended use is not permitted by statutory regulation or exceeds the permitted use, you will need to obtain permission directly from the copyright holder. To view a copy of this licence, visit http://creativecommons.org/licenses/by/4.0/. 
homotopy analysis method and nonpolynomial splines technique for solving ninth order BVPs. Siddiqi et al. $[11,12]$ used spline technique for solving linear tenth and twelfth order BVPs.

For the past few decades, wavelet based numerical methods have gained great importance for solution of HOBVPs, because of their ease in implementation. In literature, there exist several wavelet families. Amongst different kinds, Haar wavelet (HW) deserves special attention. HW is based on Haar functions which were defined by the Hungarian mathematician Alfred Haar [13] in 1909. For the first time these wavelets were used for the problems of calculus in 1997. HW comprises rectangular functions which are discontinuous at the endpoints of the interval. Therefore, HW is not directly implementable for solution of differential equations. To remove this ambiguity, Cattani [14] used spline approach to regularize these wavelets. Another way is to approximate highest order derivative by HW series. This approach was investigated by Chen and Hsiao [15, 16]. Later on, this approach became popular and has been applied to different problems. In this direction, Lepik [17] presented the solution of a higher order differential equation by using HW. Fazal et al. [18, 19] and Reddy et al. [20] solved fourth, fifth, sixth, seventh, and ninth order boundary value problems using HW. Umer [20] implemented the HW technique for solving heat convection radiation problems. Agarwal et al. [21, 22] investigated some special functions in the analysis of differential equations and some existences and uniqueness results for fractional nonlocal thermistor problem. Chu [23] and his collaborators studied fractional multi-dimensional Navier-Stokes equation. Sunarto et al. [24] implemented iterative method for solving one-dimensional fractional mathematical physics model via quarter-sweep and PAOR. The authors in $[25,26]$ explored global exponential stability of Clifford-valued neural networks and new quantum boundaries for quantum Simpson's and quantum Newton's type inequalities for preinvex functions respectively. Rezapour et al. [27] used DGJIM and ADM methods for multi-term fractional BVP involving the generalized $\psi$-RL-operators.

The rest of the paper is organized in the following way. In Sect. 2, basic definitions of HW are described. Method description and convergence are given in Sects. 3 and 4 respectively. Test problems are presented in Sect. 5, while conclusion is addressed in Sect. 6.

\subsection{Motivation}

The main motivation of this work is to develop HW based algorithm for solving HOBVPs. Also we will elaborate the scheme coupled with quasilinearization to tackle nonlinearity in tenth, twelfth, and thirteenth order BVPs. Convergence result will also be a part of this work.

\section{Haar wavelet and its integrals}

Suppose $w \in[c, d)$, where $c$ and $d$ are constants. We define $\mathbb{M}=2^{J}$, where J represents the maximal resolution level. Further subdivide $[c, d)$ into $2 \mathbb{M}$ subintervals with mesh size $\Delta w=\frac{1}{2 \mathbb{M}}(d-c)$. Next define two parameters, namely dilation $J=0,1,2,3, \ldots, \mathrm{J}$, and translation parameter $k=0,1,2, \ldots, m-1$. These parameters show the integer decomposition of wavelet number $i=k+m+1$, where $m=2^{j}$. Then $i$ th Haar wavelet is defined as follows 
$[17,21]:$

$$
\mathbb{H}_{i}(w)= \begin{cases}1 & \text { if } \alpha_{1}(i) \leq w<\alpha_{2}(i) \\ -1 & \text { if } \alpha_{2}(i) \leq w<\alpha_{3}(i) \\ 0 & \text { elsewhere }\end{cases}
$$

where $\alpha_{s+1}(i)=c+(2 k+s) \eta \Delta w, s=0,1,2$, and $\eta=2^{J-j}$. Equation (1) is valid if $i>2$. For $i=1$, the scaling function is defined as

$$
\mathbb{H}_{1}(w)= \begin{cases}1 & \text { if } w \in[c, d) \\ 0 & \text { elsewhere }\end{cases}
$$

For $i=2$, the mother wavelet is given by

$$
\left\{\begin{array}{l}
\alpha_{1}(2)=c \\
\alpha_{2}(2)=\frac{1}{2}(d-c) \\
\alpha_{3}(2)=d
\end{array}\right.
$$

To solve $n$th order BVPs, one needs repeated integrals of the form

$$
\mathbb{P}_{i, \mu}(w)=\int_{c}^{w} \int_{c}^{w} \int_{c}^{w} \cdots \int_{c}^{w} \mathbb{H}_{i}(s) d s^{\mu}=\frac{1}{(\mu-1) !} \int_{c}^{w}(w-s)^{\mu-1} \mathbb{H}_{i}(s) d s,
$$

where $i=1,2,3, \ldots, 2 \mathbb{M}$ and $\mu=1,2, \ldots, n$. Using Eq. (1) and Eq. (2), the closed form expression of these integrals is given by [17]:

$$
\mathbb{P}_{i, \mu}(w)= \begin{cases}0, & \text { if } w<\alpha_{1}(i), \\ \frac{1}{\mu !}\left\{w-\alpha_{1}(i)\right\}^{\mu} & \text { if } \alpha_{1}(i) \leq w<\alpha_{2}(i), \\ \frac{1}{\mu !}\left[\left\{w-\alpha_{1}(i)\right\}^{\mu}-2\left\{w-\alpha_{2}(i)\right\}^{\mu}\right] & \text { if } \alpha_{2}(i) \leq w<\alpha_{3}(i), \\ \frac{1}{\mu !}\left[\left\{w-\alpha_{1}(i)\right\}^{\mu}-2\left\{\left(w-\alpha_{2}(i)\right\}^{\mu}+\left\{w-\alpha_{3}(i)\right\}^{\mu}\right]\right. & \text { if } w>\alpha_{3}(i) .\end{cases}
$$

Equation (5) is valid for $i \geq 2$, when $i=1$ then

$$
\mathbb{P}_{1, \mu}(w)=\frac{1}{\mu !}(w-c)^{\mu} .
$$

The proposed method is based on collocation approach, therefore the collocation points are

$$
w_{p}=\frac{p-0.5}{2 \mathbb{M}}, \quad p=1,2,3, \ldots .2 \mathbb{M}
$$

\section{Method description for linear problems}

This section is devoted to describing the proposed methodology for solving HOBVPs. For this purpose consider the $n$th order ordinary differential equations

$$
\mathfrak{L} \Psi(w)=g(w), \quad w \in \Omega=[\mathcal{C}, \mathcal{D}],
$$


where $\mathfrak{L}$ is a general differential operator and $g$ is a known function. The corresponding initial and boundary conditions are

$$
\Psi^{(\sigma)}(\mathcal{C})=A_{\sigma}, \quad \sigma=0,1,2, \ldots, n-1,
$$

or

$$
\Psi^{(\sigma-1)}(\mathcal{C})=A_{\sigma}, \quad \Psi^{(\sigma-1)}(\mathcal{D})=B_{\sigma}, \quad \sigma=1,2,3, \ldots, \frac{n}{2},
$$

or

$$
\Psi^{(\sigma-2)}(\mathcal{C})=A_{\sigma}, \quad \Psi^{(\sigma-2)}(\mathcal{D})=B_{\sigma}, \quad \sigma=2,4,6, \ldots, n
$$

Here, we present the method only for initial conditions, boundary conditions will be treated later. Next we approximate the highest order derivative by truncated HW series as follows:

$$
\Psi^{(n)}(w)=\sum_{i=1}^{2 \mathbb{M}} b_{i} \mathbb{H}_{i}(w),
$$

where $b_{i}$ stands for unknown wavelet coefficients to be calculated. Integrating Eq. (12), $n-\sigma$ times in the domain $[\mathcal{C}, w]$, we have

$$
\Psi^{(\sigma)}(w)=\sum_{i=1}^{2 \mathbb{M}} b_{i} \mathbb{P}_{i, n-\sigma}(w)+\sum_{\beta=0}^{n-\sigma-1} \frac{1}{\beta !}(w-\mathcal{C})^{\beta} \Psi^{(\sigma+\beta)}(\mathcal{C}) .
$$

Now inserting Eq. (7) and (12)-(13) in Eq. (8) leads to the system of $2 \mathbb{M}$ equations with $2 \mathbb{M}$ unknowns $b_{i}$. After computing $b_{i}$, the desired solution can be extracted from Eq. (13) by putting $\sigma=0$. It is to be noted that the proposed method requires initial conditions of the form $\Psi^{(\sigma)}(\mathcal{C}), \sigma=0,1,2, \ldots, n-1$. If conditions $\Psi^{(s)}\left(w_{r}\right)=\Gamma_{r}$, where $w_{r} \in[\mathcal{C}, \mathcal{D}]$, and $s<n$ is any nonnegative integer are known instead of $\Psi^{(s)}(\mathcal{C})$, we need to find $\Psi^{(s)}(\mathcal{C})$. By doing this one can write Eq. (13) as follows:

$$
\Gamma_{r}=\sum_{i=1}^{2 \mathbb{M}} b_{i} \mathbb{P}_{i, n-\sigma}(r)+\sum_{\beta=0}^{n-\sigma-1} \frac{1}{\beta !}(r-c)^{\beta} \Psi^{(\sigma+\beta)}(\mathcal{C}) .
$$

Equation (14) denotes the system of linear equations from which the rest of initial conditions can be computed.

\subsection{Nonlinear case and quasilinearization}

Consider a nonlinear $n$th order differential equation of the form

$$
\Psi^{(n)}(w)=g\left(w, \Psi, \Psi^{(1)}, \Psi^{(2)}, \ldots, \Psi^{(n-1)}\right), \quad w \in \Omega,
$$

with initial and boundary conditions

$$
\Psi^{(\sigma-1)}(w)=\mu_{\sigma},
$$


where $\Psi^{(\sigma)}$ represents $\sigma$ th derivative of $\Psi, \mu_{\sigma}$ are given constants. Using the quasilinearization procedure [28], Eq. (15) reduces to

$$
\Psi_{\gamma+1}^{(n)}=g+\sum_{\sigma=0}^{n-1}\left(\Psi_{\gamma+1}^{(\sigma)}-\Psi_{\gamma}^{(\sigma)}\right) \frac{\partial g}{\partial \Psi_{\gamma}^{(\sigma)}} .
$$

In Eq. (17) $\gamma$ stands for the number of iterations. It is clear from the above equation that the value of $\Psi$ at the $(\gamma+1)$ th iteration can be computed if it is known at the $\gamma$ th iteration.

\section{Convergence analysis}

In this section, we discuss the convergence of 10th order problem. For this purpose we consider the given problem

$$
G\left(w, \Psi, \Psi^{(1)}, \Psi^{(2)}, \Psi^{(3)}, \Psi^{(4)}, \Psi^{(5)}, \Psi^{(6)}, \Psi^{(7)}, \Psi^{(8)}, \Psi^{(9)}, \Psi^{(10)}\right)=0 .
$$

Assume that

$$
\Psi^{(10)}(w)=\sum_{i=1}^{\infty} b_{i} \mathbb{H}_{i}(w)
$$

Alternatively this series can be written as

$$
\Psi^{(10)}(w)=b_{1} \mathbb{H}_{1}+\sum_{j=0}^{\infty} \sum_{\ell=0}^{2^{j}-1} b_{2 j+\ell+1} \mathbb{H}_{2 j+\ell+1}(w),
$$

where $i=m+\ell+1, j=0,1,2, \ldots J, \ell=0,1,2, \ldots m-1$. Integrating Eq. (19) ten times the resultant is

$$
\Psi(w)=\frac{b_{1}}{10 !}+\sum_{j=0}^{\infty} \sum_{\ell=0}^{2^{j}-1} b_{2^{j}+\ell+1} \mathbb{P}_{2^{j}+\ell+1}, 10(w)+\mathbb{B}(w),
$$

where $\mathbb{B}(w)$ denotes boundary terms. To express the convergence as a theorem, we use the following lemma.

Lemma 4.1 ([29]) If $\Psi(w) \in L^{2}(R)$ with $\left|\Psi^{\prime}(w)\right| \leq \rho$ for all $w \in(0,1)$ and $\Psi(w)=$ $\sum_{i=0}^{\infty} b_{i} \mathbb{H}_{i}(w)$, then $\left|b_{i}\right| \leq \frac{\rho}{2^{j+1}}$.

Theorem 4.2 If $\Psi$ and $\Psi_{2 \mathbb{M}}$ are the exact and approximate solutions of Eq. (18), then the error norm at $J$ resolution level is given by

$$
\left\|E_{J}\right\|=O\left[\left(\frac{1}{2^{J+1}}\right)^{2}\right]
$$

Proof From Eqs. (19) and (21) we have

$$
\left|E_{\mathbb{M}}\right|=\left|\Psi(w)-\Psi_{\mathbb{M}}(w)\right|=\left|\sum_{j=J+1}^{\infty} \sum_{\ell=0}^{2^{j}-1} b_{2 j+\ell+1} \mathbb{P}_{2 j+\ell+1}, 10(w)\right| .
$$


Using the definition of error norm, one can write

$$
\begin{aligned}
& \left\|E_{\mathbb{M}}\right\|_{2}^{2}=\int_{0}^{1}\left(\sum_{j=J+1}^{\infty} \sum_{\ell=0}^{2^{j}-1} b_{2^{j}+\ell+1} \mathbb{P}_{2^{j}+\ell+1}, 10\right. \\
& \\
& \left\|E_{\mathbb{M}}\right\|_{2}^{2}=\sum_{j=J+1}^{\infty} \sum_{\ell=0}^{2^{j}-1} \sum_{s=J+1}^{\infty} \sum_{t=0}^{2^{t}-1} b_{2^{j}+\ell+1} b_{2^{s}+t+1} \int_{0}^{1} \mathbb{P}_{2^{j}+\ell+1}, 10(w)
\end{aligned}
$$

Using the fact that Haar wavelet integrals are monotonically increasing functions [29] coupled with Lemma 4.1, the resultant is

$$
\begin{aligned}
\left\|E_{\mathbb{M}}\right\|_{2}^{2} \leq & \sum_{j=J+1}^{\infty} \sum_{\ell=0}^{2^{j}-1} \sum_{s=J+1}^{\infty} \sum_{t=0}^{2^{t}-1} \frac{\rho}{2^{j+1}} \frac{\rho}{2^{s+1}}\left\{\frac{1}{40,320}\left(\frac{1}{2^{j+1}}\right)^{2}+\frac{1}{1440}\left(\frac{1}{2^{j+1}}\right)^{4}\right. \\
& \left.+\frac{1}{8640}\left(\frac{1}{2^{j+1}}\right)^{6}+\frac{1}{40,320}\left(\frac{1}{2^{j+1}}\right)^{8}+\frac{1}{1,814,400}\left(\frac{1}{2^{j+1}}\right)^{10}\right\} \\
& \times\left\{\frac{1}{40,320}\left(\frac{1}{2^{s+1}}\right)^{2}+\frac{1}{1440}\left(\frac{1}{2^{s+1}}\right)^{4}+\frac{1}{8640}\left(\frac{1}{2^{s+1}}\right)^{6}\right. \\
& \left.+\frac{1}{40,320}\left(\frac{1}{2^{s+1}}\right)^{8}+\frac{1}{1,814,400}\left(\frac{1}{2^{s+1}}\right)^{10}\right\}
\end{aligned}
$$

which can be written as follows:

$$
\begin{aligned}
\left\|E_{\mathbb{M}}\right\|_{2} \leq & \frac{\alpha}{17,280}\left\{\frac{1}{14}\left(\frac{1}{2^{J+1}}\right)^{2}+\frac{1}{15}\left(\frac{1}{2^{J+1}}\right)^{4} \frac{1}{65}\left(\frac{1}{2^{J+1}}\right)^{6}+\frac{1}{1190}\left(\frac{1}{2^{J+1}}\right)^{8}\right. \\
& \left.+\frac{1}{214,830}\left(\frac{1}{2^{J+1}}\right)^{10}\right\} .
\end{aligned}
$$

Hence

$$
\left\|E_{\mathbb{M}}\right\|_{2}=O\left[\left(\frac{1}{2^{J+1}}\right)^{2}\right] .
$$

From (27) it is obvious that the error norm is inversely proportional to the resolution level. By increasing the resolution level the error norm should decrease. The same criteria can be applied for the convergence of other HOBVPs.

\section{Numerical examples and discussion}

In this section, the proposed method is applied to solve 10th, 12th, and 13th order BVPs. To check the performance and reliability of the method, the computed solutions are matched with the exact ones and those presented before.

\subsection{Example}

Consider the 10th order linear boundary value problem [30]

$$
\Psi^{(10)}(w)=-8 e^{w}+\Psi^{(2)}(w), \quad w \in[0,1]
$$


with initial and boundary conditions

$$
\begin{cases}\Psi^{(\sigma)}(0)=1-\sigma & \text { where } \sigma=0,1,2,3,4 \\ \Psi^{(\sigma)}(1)=-\sigma e & \text { where } \sigma=0,1,2,3,4\end{cases}
$$

The analytic solution of this problem is given by

$$
\Psi(w)=(1-w) e^{w} .
$$

Using the method discussed earlier, we have

$$
\Psi^{(10)}(w)=\sum_{i=1}^{2 \mathbb{M}} b_{i} \mathbb{H}_{i}(w)
$$

Integrating Eq. (31) ten times, we get

$$
\Psi^{(\sigma)}(w)=\sum_{i=1}^{2 \mathbb{M}} b_{i} \mathbb{P}_{i, 10-\sigma}(w)+\sum_{\beta=0}^{9-\sigma} \frac{1}{\beta !}(w)^{\beta} \Psi^{(\sigma+\beta)}(0), \quad \text { where } \sigma=0,1, \ldots, 9 .
$$

In Eq. (29) five initial conditions are given, we compute the remaining five initial conditions using Eq. (29) in Eq. (32) as follows:

$$
\begin{aligned}
\Psi^{(5)}(0)= & -4.0005+\sum_{i=1}^{2 \mathbb{M}} b_{i}\left[-15,120 \mathbb{P}_{i, 10}(1)+6720 \mathbb{P}_{i, 9}(1)-1260 \mathbb{P}_{i, 8}(1)\right. \\
& \left.+120 \mathbb{P}_{i, 7}(1)-5 \mathbb{P}_{i, 6}(1)\right], \\
\Psi^{(6)}(0)= & -4.98528+\sum_{i=1}^{2 \mathbb{M}} b_{i}\left[302,400 \mathbb{P}_{i, 10}(1)-141,120 \mathbb{P}_{i, 9}(1)+2772 \mathbb{P}_{i, 8}(1)\right. \\
& \left.-2760 \mathbb{P}_{i, 7}(1)+120 \mathbb{P}_{i, 6}(1)\right], \\
\Psi^{(7)}(0)= & -6.19998+\sum_{i=1}^{2 \mathbb{M}} b_{i}\left[-272,160 \mathbb{P}_{i, 10}(1)+13,104 \mathbb{P}_{i, 9}(1)-267,120 \mathbb{P}_{i, 8}(1)\right. \\
& \left.+27,720 \mathbb{P}_{i, 7}(1)-1260 \mathbb{P}_{i, 6}(1)\right], \\
\Psi^{(8)}(0)= & -5.47661+\sum_{i=1}^{2 \mathbb{M}} b_{i}\left[+127,008 \mathbb{P}_{i, 10}(1)-62,496 \mathbb{P}_{i, 9}(1)+131,040 \mathbb{P}_{i, 8}(1)\right. \\
& \left.-141,120 \mathbb{P}_{i, 7}(1)+6720 \mathbb{P}_{i, 6}(1)\right], \\
\Psi^{(9)}(0)= & -14.2157+\sum_{i=1}^{2 \mathbb{M}} b_{i}\left[-2,540,160 \mathbb{P}_{i, 10}(1)+127,008 \mathbb{P}_{i, 9}(1)-272,160 \mathbb{P}_{i, 8}(1)\right. \\
& \left.+302,400 \mathbb{P}_{i, 7}(1)-15,120 \mathbb{P}_{i, 6}(1)\right] .
\end{aligned}
$$


Table 1 Comparison of approximate and exact solution and absolute error of Example 5.1 when $J=4$

\begin{tabular}{lllll}
\hline$w$ & Approximate solution & Exact solution & Error & {$[30]$} \\
\hline 0.0 & 1.000000000000000 & 1.000000000000000 & $0.00000 \mathrm{e}-00$ & $0.00000 \mathrm{e}-00$ \\
0.1 & 0.994653826267967 & 0.994653826268083 & $1.15796 \mathrm{e}-13$ & $8.82148 \mathrm{e}-06$ \\
0.2 & 0.977122206525615 & 0.977122206528136 & $2.52120 \mathrm{e}-12$ & $8.64267 \mathrm{e}-06$ \\
0.3 & 0.944901165290643 & 0.944901165303202 & $1.25587 \mathrm{e}-11$ & $2.92062 \mathrm{e}-06$ \\
0.4 & 0.895094818551454 & 0.895094818584762 & $3.33081 \mathrm{e}-11$ & $5.96046 \mathrm{e}-07$ \\
0.5 & 0.824360635288676 & 0.824360635350064 & $6.13880 \mathrm{e}-11$ & $6.73532 \mathrm{e}-06$ \\
\hline
\end{tabular}

Using collocation points $w_{p}$ (see Eq. (7)) and initial conditions, we obtain

$$
\begin{aligned}
& \sum_{i=1}^{2 \mathbb{M}} b_{i}\left[\mathbb{H}_{i}\left(w_{p}\right)-\mathbb{P}_{i, 8}\left(w_{p}\right)-A_{1} \mathbb{P}_{i, 10}(1)-A_{2} \mathbb{P}_{i, 9}(1)\right. \\
& \left.-A_{3} \mathbb{P}_{i, 8}(1)-A_{4} \mathbb{P}_{i, 7}(1)-A_{5} \mathbb{P}_{i, 6}(1)\right]=-8 e^{w_{p}}+A_{6},
\end{aligned}
$$

where

$$
\begin{aligned}
& \left\{\begin{array}{l}
A_{1}=-2520 w_{p}^{3}+12,600 w_{p}^{4}-2268 w_{p}^{5}+17,640 w_{p}^{6}-5040 w_{p}^{7}, \\
A_{2}=1120 w_{p}^{3}-5880 w_{p}^{4}+10,920 w_{p}^{5}-8680 w_{p}^{6}+2520 w_{p}^{7} \\
A_{3}=-210 w_{p}^{3}+1155 w_{p}^{4}-2226 w_{p}^{5}+1820 w_{p}^{6}-540 w_{p}^{7} \\
A_{4}=20 w_{p}^{3}-115 w_{p}^{4}+231 w_{p}^{5}-196 w_{p}^{6}+60 w_{p}^{7}
\end{array}\right. \\
& \left\{\begin{array}{l}
A_{5}=-0.8333 w_{p}^{3}+5 w_{p}^{4}-10.5 w_{p}^{5}+9.3333 w_{p}^{6}-3 w_{p}^{7}, \\
A_{6}=-1-2 w_{p}-1.5 w_{p}^{2}+161.83 w_{p}^{3}-587.7 w_{p}^{4}+892.4 w_{p}^{5}-623.00 w_{p}^{6}-164.9 w_{p}^{7}
\end{array}\right.
\end{aligned}
$$

Equation (33) represents $2 \mathbb{M}$ equations and $2 \mathbb{M}$ unknowns (wavelets coefficients). After calculating the unknowns, approximate solution can be obtained from Eq. (32) when $\sigma=$ 0 . In Table 1, we matched computed solutions with the exact solution and those presented in [30]. The same table also addresses absolute errors. It is obvious from the table that the proposed method gives better results than [30], which shows betterment of the proposed method. Solution profiles of exact and approximate solutions together with absolute error are shown in Fig. 1. From the figure it can be seen that exact and approximate solutions are in good agreement.

\subsection{Example}

Consider the 10th order nonlinear boundary value problem [30]

$$
\Psi^{(10)}(w)=e^{-w} \Psi^{2}(w), \quad 0 \leq w \leq 1,
$$

subject to the following conditions:

$$
\begin{cases}\Psi^{(\sigma)}(0)=1 & \sigma=0,2,4,6,8 \\ \Psi^{(\sigma)}(1)=e & \sigma=0,2,4,6,8\end{cases}
$$

The exact solution is

$$
\Psi(w)=e^{w} .
$$



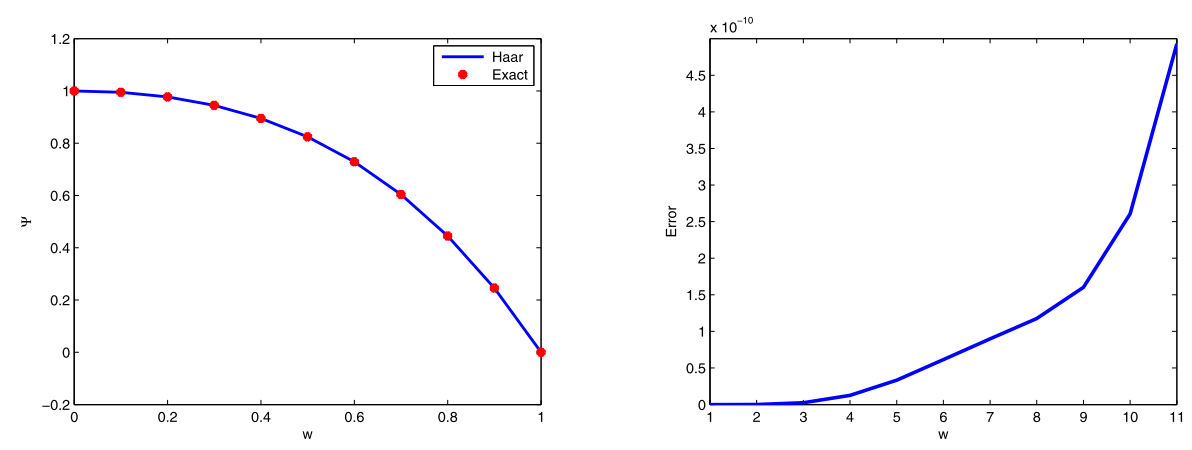

Figure 1 Solution profile of Example 5.1 for $J=4$

Applying quasilinearization, Eq. (34) and Eq. (35) take the form

$$
\begin{aligned}
& \Psi_{\gamma+1}^{(10)}(w)-2 e^{-w} \Psi_{\gamma+1}(w) \Psi_{\gamma}(w)=-e^{-w} \Psi_{\gamma}^{2}(w), \\
& \Psi_{\gamma+1}^{(\sigma)}(0)=1, \quad \sigma=0,2,4,6,8, \\
& \Psi_{\gamma+1}^{(\sigma)}(1)=e, \quad \sigma=0,2,4,6,8 .
\end{aligned}
$$

Approximating the highest order derivative in Eq. (37), we have

$$
\Psi_{\gamma+1}^{(10)}(w)=\sum_{i=1}^{2 \mathbb{M}} b_{i} \mathbb{H}_{i}(w) .
$$

Integrating Eq. (40) ten times leads to

$$
\Psi_{\gamma+1}^{(\sigma)}(w)=\sum_{i=1}^{2 \mathbb{M}} b_{i} \mathbb{P}_{i, 10-\sigma}(w)+\sum_{\beta=0}^{9-\sigma} \frac{1}{\beta !}(w)^{\beta} \Psi_{\gamma+1}^{(\sigma+\beta)}(0), \quad \sigma=0,1, \ldots, 9 .
$$

Using Eq. (7) and Eq. (41), we get

$$
\begin{aligned}
\sum_{i=1}^{2 \mathbb{M}} b_{i} & {\left[\mathbb{H}_{i}\left(w_{p}\right)-2 e^{-w_{p}} \Psi_{\gamma}\left\{\mathbb{P}_{i, 10}\left(w_{p}\right)-w_{p} \mathbb{P}_{i, 10}(1)+A_{1} \mathbb{P}_{i, 8}(1)+A_{2} \mathbb{P}_{i, 6}(1)\right.\right.} \\
& \left.\left.+A_{3} \mathbb{P}_{i, 4}(1)+A_{4} \mathbb{P}_{i, 2}(1)\right\}\right] \\
= & -e^{-w_{p}} \Psi_{\gamma}^{2}\left(w_{p}\right)+2 \Psi_{\gamma}\left(w_{p}\right)\left(A_{5}+A_{6}\right),
\end{aligned}
$$

where

$$
\left\{\begin{array}{l}
A_{1}=\frac{1}{40,320} w_{p}-\frac{1}{6} w_{p}^{3}, \\
A_{2}=\frac{-7}{360} w_{p}+\frac{1}{36} w_{p}^{3}-\frac{1}{120} w_{p}^{5}, \\
A_{3}=\frac{31}{15,120} w_{p}-\frac{7}{2160} w_{p}^{3}+\frac{1}{720} w_{p}^{5}-\frac{1}{5040} w_{p}^{7}, \\
A_{4}=\frac{-127}{604,800} w_{p}+\frac{31}{90,720} w_{p}^{3}-\frac{7}{43,200} w_{p}^{5}+\frac{1}{30,240} w_{p}^{7}-\frac{1}{362,880} w_{p}^{9}, \\
A_{5}=1+1.0000720 w_{p}+\frac{1}{2} w_{p}^{2}+\frac{1}{24} w_{p}^{4}+\frac{1}{720} w_{p}^{6}+\frac{1}{40,320} w_{p}^{8}, \\
A_{6}=-0.1665482710 w_{p}^{3}+8.39149 \times 10^{-3} w_{p}^{5}+1.849 \times 10^{-4} w_{p}^{7}+4.7351 \times 10^{-6} w_{p}^{9} .
\end{array}\right.
$$


Table 2 Comparison of approximate and exact solution and absolute error of Example 5.2 when $J=4$

\begin{tabular}{lllll}
\hline$w$ & Approximate & Exact & Error & {$[30]$} \\
\hline 0.0 & 1.00000 & 1.00000 & $0.00 \mathrm{e}-00$ & $0.00 \mathrm{e}-00$ \\
0.1 & 1.10517 & 1.10517 & $7.08 \mathrm{e}-06$ & $1.25 \mathrm{e}-06$ \\
0.2 & 1.22141 & 1.22140 & $1.34 \mathrm{e}-05$ & $8.69 \mathrm{e}-06$ \\
0.3 & 1.34987 & 1.34985 & $1.85 \mathrm{e}-05$ & $2.14 \mathrm{e}-05$ \\
0.4 & 1.49184 & 1.49182 & $2.18 \mathrm{e}-05$ & $4.35 \mathrm{e}-06$ \\
0.5 & 1.64874 & 1.64872 & $2.29 \mathrm{e}-05$ & $3.96 \mathrm{e}-05$ \\
0.6 & 1.82214 & 1.82211 & $2.17 \mathrm{e}-05$ & $5.40 \mathrm{e}-05$ \\
0.7 & 2.01377 & 2.01375 & $1.84 \mathrm{e}-05$ & $6.79 \mathrm{e}-05$ \\
\hline
\end{tabular}
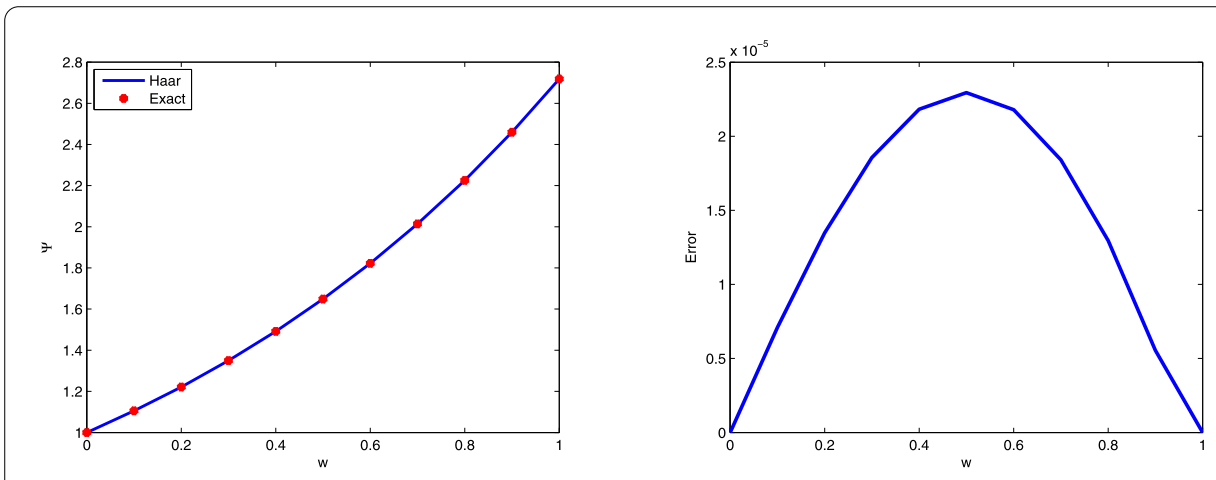

Figure 2 Solution profile of Example 5.2 for $J=4$

Equation (42) contains a total of $2 \mathbb{M}$ equations in $2 \mathbb{M}$ unknowns. The system has been solved for unknowns with initial approximation $\Psi_{0}(w)=1$. After calculation of $b_{i}$, approximate solutions have been calculated from Eq. (38) for $\sigma=0$. In Table 2 the computed solutions are compared with the exact solution and the results given in [30]. In the same table we also recorded the absolute error. From the table one can see that the proposed method has comparatively good results. In Fig. 2 the plots of exact, approximate solutions and absolute errors are presented. The figure shows that exact and approximate solutions premise well.

\subsection{Example}

Consider the 12th order linear boundary value problem [31]

$$
\Psi^{(12)}(w)+w \Psi(w)=-e^{w}\left(120+23 w+w^{3}\right), \quad 0 \leq w \leq 1,
$$

subject to the following conditions:

$$
\begin{cases}\Psi^{(\sigma)}(0)=\sigma(2-\sigma), & \sigma=0,1,2,3,4,5 \\ \Psi^{(\sigma)}(1)=-\sigma^{2} e, & \sigma=0,1,2,3,4,5\end{cases}
$$

The exact solution of this problem is

$$
\Psi(w)=w(1-w) e^{w} .
$$


Table 3 Comparison of approximate and exact solution and absolute error of Example 5.3 when $J=4$

\begin{tabular}{lllll}
\hline$w$ & Approximate solution & Exact solution & Error & {$[31]$} \\
\hline 0.0 & 0.000000000000000 & 0.0000000000000000 & $0.00 \mathrm{e}-0$ & $0.00 \mathrm{e}-00$ \\
0.1 & 0.099465382626784 & 0.099465382626808 & $2.38 \mathrm{e}-14$ & $3.00 \mathrm{e}-11$ \\
0.2 & 0.195424441303850 & 0.195424441305627 & $1.77 \mathrm{e}-12$ & $0.10 \mathrm{e}-10$ \\
0.3 & 0.283470349535050 & 0.283470349590961 & $5.59 \mathrm{e}-11$ & $1.00 \mathrm{e}-10$ \\
0.4 & 0.358037926531124 & 0.358037927433905 & $9.02 \mathrm{e}-11$ & $2.00 \mathrm{e}-10$ \\
0.5 & 0.412180309409399 & 0.412180317675032 & $8.26 \mathrm{e}-11$ & $1.10 \mathrm{e}-09$ \\
\hline
\end{tabular}
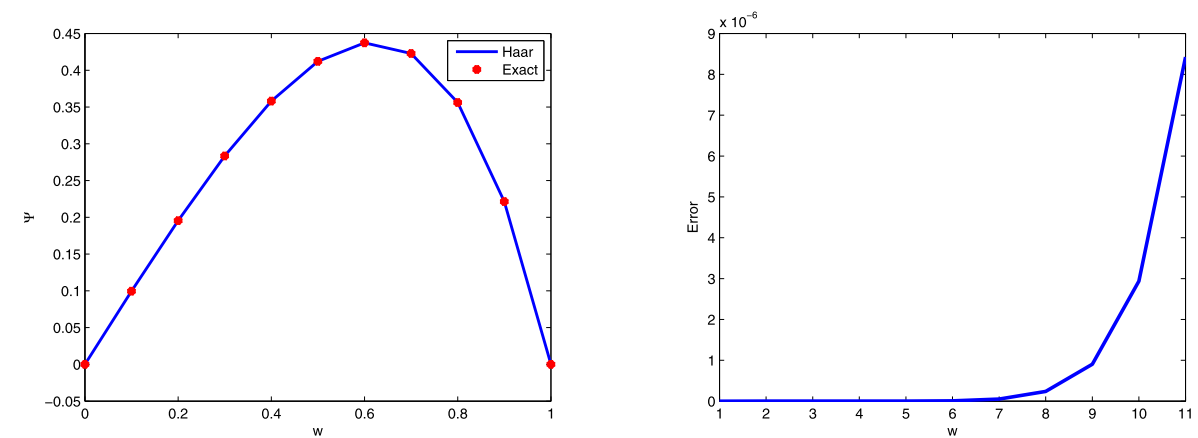

Figure 3 Solution profile of Example 5.3 for $J=4$

The approximate and exact solutions together with absolute error have been addressed in Table 3. The same table also shows the comparison of our results with those obtained via the homotopy perturbation method addressed in [31]. It has been observed that our results are better. Solution profiles of exact versus approximate solutions coupled with absolute error are shown in Fig. 3. From the figure it is clear that both solutions are in good agreement.

\subsection{Example}

Consider the 12th order nonlinear boundary value problem [32]

$$
\Psi^{(12)}(w)=2 e^{w} \Psi^{2}(w)+\Psi^{(3)}(w), \quad 0 \leq w \leq 1,
$$

with boundary conditions

$$
\begin{cases}\Psi^{(2 \sigma)}(0)=1, & \sigma=0,1,2,3,4,5, \\ \Psi^{(2 \sigma)}(1)=e^{-1}, & \sigma=0,1,2,3,4,5 .\end{cases}
$$

The exact solution is

$$
\Psi(w)=e^{-w}
$$

The problem is nonlinear which has been linearized using quasilinearization. Computed solutions have been matched with the existing results [32] in Table 4. The same table contains the absolute error. From the table, it is obvious that the computed results are comparatively better. In Fig. 4, the plots of approximate, exact solutions and error are shown, which shows that the approximate and exact solution are close to each other. 
Table 4 Comparison of approximate and exact solution and absolute error of Example 5.4 when $J=4$

\begin{tabular}{lllll}
\hline$w$ & Approximate solution & Exact solution & Error & {$[32]$} \\
\hline 0.0 & 1.000000 & 1.0000000 & $0.00 \mathrm{e}-0$ & $0.00 \mathrm{e}-0$ \\
0.1 & 0.904837 & 0.9048374 & $2.64 \mathrm{e}-7$ & $2.64 \mathrm{e}-7$ \\
0.2 & 0.818730 & 0.8187307 & $5.02 \mathrm{e}-7$ & $5.03 \mathrm{e}-7$ \\
0.3 & 0.740817 & 0.7408182 & $6.92 \mathrm{e}-7$ & $6.92 \mathrm{e}-7$ \\
0.4 & 0.670319 & 0.6703200 & $8.13 \mathrm{e}-7$ & $8.14 \mathrm{e}-7$ \\
0.5 & 0.6065298 & 0.6065306 & $8.55 \mathrm{e}-7$ & $8.55 \mathrm{e}-7$ \\
\hline
\end{tabular}
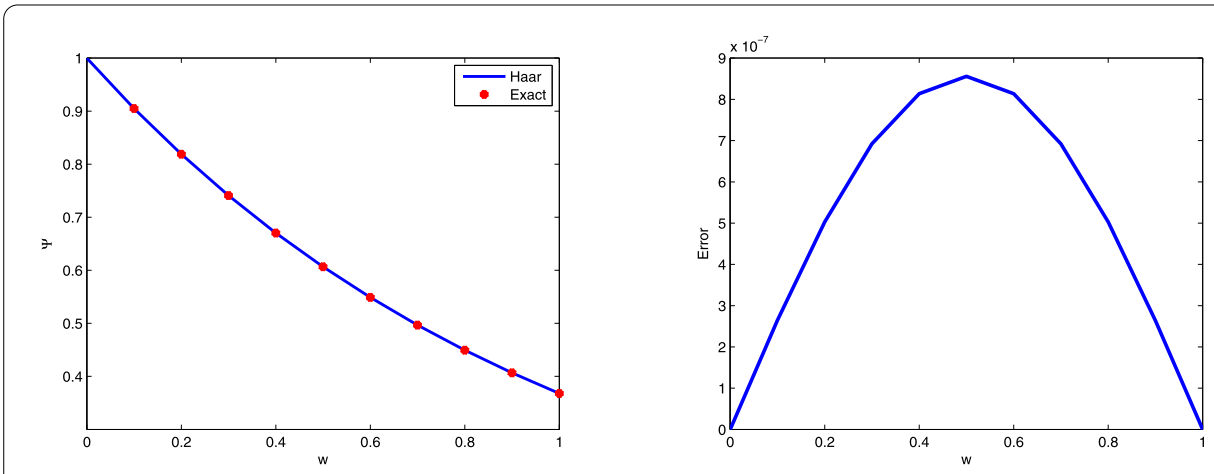

Figure 4 Solution profile of Example 5.4 for $J=4$

\subsection{Example}

Consider the 13th order linear boundary value problem [33]

$$
\Psi^{(13)}(w)=\cos (w)-\sin (w), \quad w \in[0,1]
$$

with boundary conditions

$$
\begin{cases}\Psi^{(\sigma)}(0)=1, \quad \sigma=0,1,4,5, & \\ \Psi^{(\sigma)}(0)=1, \quad \sigma=2,3,6, & \\ \Psi^{(\sigma)}(1)=\cos (1)+\sin (1), & \sigma=0,4 \\ \Psi^{(\sigma)}(1)=\cos (1)-\sin (1), & \sigma=1,5 \\ \Psi^{(\sigma)}(1)=\cos (1)-\sin (1), & \sigma=2, \\ \Psi^{(\sigma)}(1)=\sin (1)-\cos (1), & \sigma=3 .\end{cases}
$$

The exact solution is

$$
\Psi(w)=\cos (w)+\sin (w) .
$$

In Table 5 we recorded the comparison of exact and calculated solutions with absolute error. The same table contains the results computed by the variational iteration method in [33]. One can see from the table that errors of present solutions are smaller. Graphical solution and absolute error are also plotted in Fig. 5, which indicates that the scheme works well for higher order problems. 
Table 5 Comparison of approximate and exact solution and absolute error of Example 5.5 when $J=4$

\begin{tabular}{lllll}
\hline$w$ & Approximate solution & Exact solution & Error & {$[33]$} \\
\hline 0.0 & 1.000000000000000 & 1.000000000000000 & $0.00000 \mathrm{e}-00$ & $0.00000 \mathrm{e}-00$ \\
0.1 & 1.094837581924854 & 1.094837581924854 & $0.00000 \mathrm{e}-00$ & $3.88578 \mathrm{e}-15$ \\
0.2 & 1.178735908636302 & 1.178735908636303 & $1.33226 \mathrm{e}-15$ & $1.46216 \mathrm{e}-13$ \\
0.3 & 1.250856695786933 & 1.250856695786946 & $1.24344 \mathrm{e}-14$ & $8.80518 \mathrm{e}-13$ \\
0.4 & 1.310479336311486 & 1.310479336311536 & $4.99600 \mathrm{e}-14$ & $2.35822 \mathrm{e}-12$ \\
0.5 & 1.357008100494460 & 1.357008100494576 & $1.15463 \mathrm{e}-13$ & $3.80140 \mathrm{e}-12$ \\
\hline
\end{tabular}
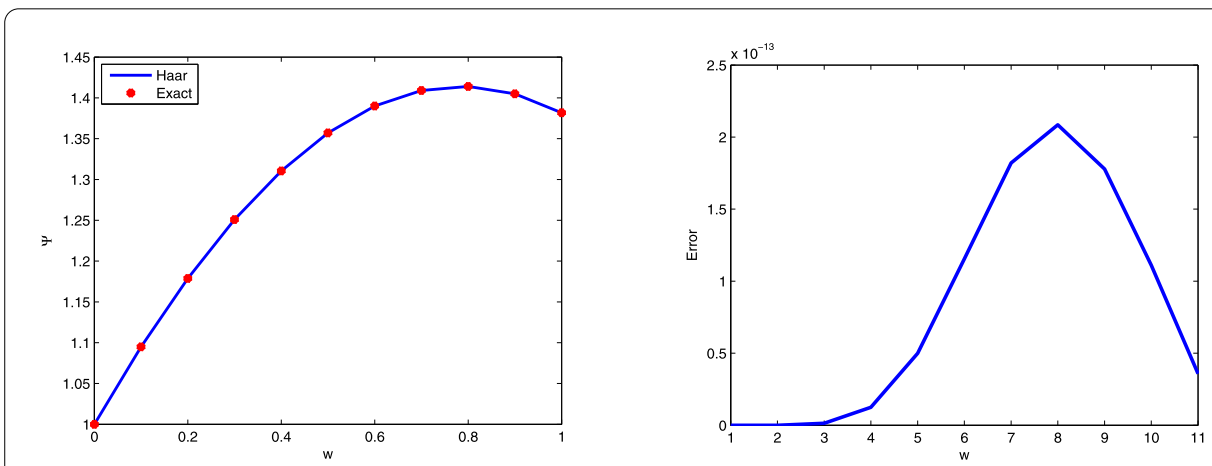

Figure 5 Solution profile of Example 5.5 for $J=4$

\subsection{Example}

Consider the 13th order nonlinear boundary value problem [33]

$$
\Psi^{(13)}(w)=e^{-w} \Psi^{2}(w), \quad 0 \leq w \leq 1,
$$

with given boundary conditions

$$
\begin{cases}\Psi^{(\sigma)}(0)=1, & \sigma=0,1,2,3,4,5,6 \\ \Psi^{(\sigma)}(1)=e, & \sigma=0,1,2,3,4,5 .\end{cases}
$$

The exact solution is

$$
\Psi(w)=e^{w} .
$$

The computed and exact solution and absolute error are displayed in Table 6. From the table it is proved that the proposed method has good results in case of nonlinear BVPs. The plot of exact, approximate solutions and error are shown in Fig. 6. From the figure one can observe the coincidence of exact and approximate solutions.

\section{Conclusion}

In this study, the Haar wavelets collocation has been applied for the numerical solution of HBVPs. The quasilinearization technique has been applied to tackle nonlinearity. Several examples have been solved using the proposed method which confirmed the efficiency and reliability. The computed results have been compared with the exact solution and those available in literature. From tabulated data and graphical solutions it has been observed 
Table 6 Comparison of approximate and exact solution and absolute error of Example 5.6 when $J=4$

\begin{tabular}{lllll}
\hline$w$ & Approximate solution & Exact solution & Error & [33] \\
\hline 0.0 & 1.000000000000000 & 1.000000000000000 & $0.00000 \mathrm{e}-00$ & $4.17444 \mathrm{e}-14$ \\
0.1 & 1.105170918075633 & 1.105170918075648 & $1.50990 \mathrm{e}-14$ & $2.64144 \mathrm{e}-12$ \\
0.2 & 1.221402758158984 & 1.221402758160170 & $1.18616 \mathrm{e}-12$ & $2.99314 \mathrm{e}-11$ \\
0.3 & 1.349858807564140 & 1.349858807576003 & $1.18636 \mathrm{e}-11$ & $1.67101 \mathrm{e}-10$ \\
0.4 & 1.491824697591858 & 1.491824697641270 & $4.94124 \mathrm{e}-11$ & $6.30955 \mathrm{e}-10$ \\
0.5 & 1.648721270576312 & 1.648721270700128 & $1.23816 \mathrm{e}-10$ & $1.84757 \mathrm{e}-09$ \\
\hline
\end{tabular}

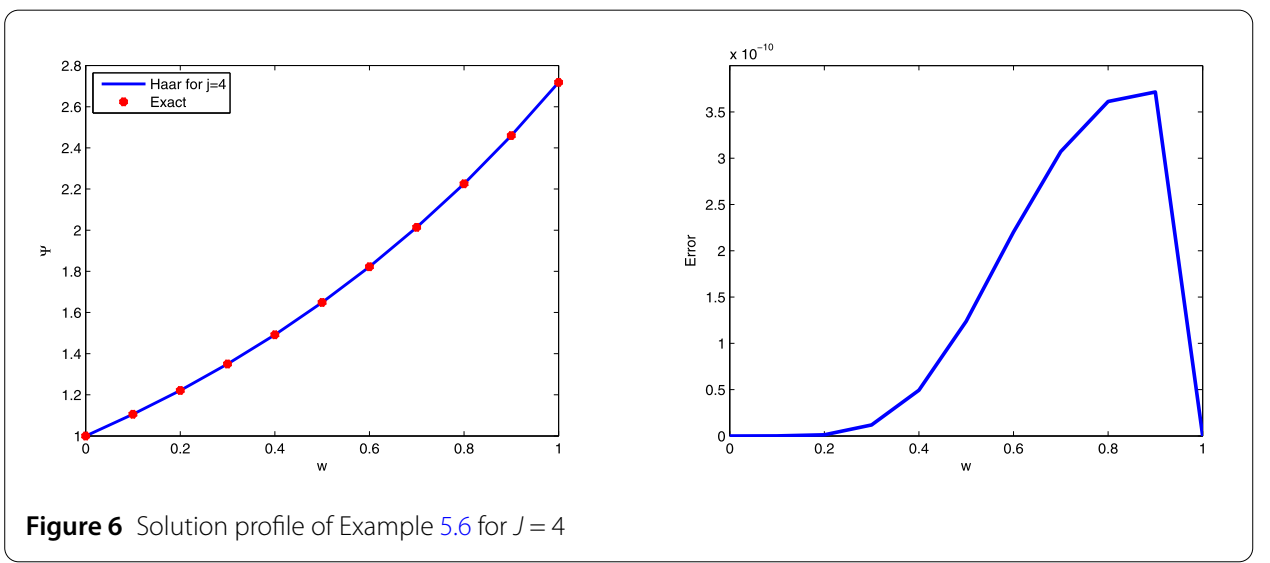

that the proposed method gives better results than quintic B-splines, homotopy perturbation method, optimal homotopy asymptotic method, and variational iteration method. In the future we will focus on extending the method for higher order partial and time fractional partial differential equations.

\section{Acknowledgements}

The authors are thankful to the anonymous reviewers for their valuable comments which improved the quality of the manuscript.

\section{Funding}

This research work is supported by the Center of Excellence in Theoretical and Computational Science (TaCS-CoE), KMUTT.

\section{Availability of data and materials}

Not applicable.

\section{Competing interests}

The authors declare that they have no competing interests.

\section{Authors' contributions}

Conceptualization, AG and SH; Methodology, SA; Software, SA; Validation, AG and AU; Formal Analysis, SA; Investigation, SH; Resources, PK; Writing-Original Draft Preparation, SA; Writing-Review and Editing, AG and AU; Visualization, PK and PC; Supervision, SH; Project Administration, PK and PC; Funding Acquisition, PK and PC. All authors read and approved the final manuscript.

\section{Author details}

${ }^{1}$ Faculty of Engineering Sciences, GIK Institute, Topi, 23640, KP, Pakistan. ${ }^{2}$ Institute of Numerical Sciences, Kohat University of Science and Technology, Kohat, 26000, KP, Pakistan. ${ }^{3}$ Department of Mathematical Sciences, University of Lakki Marwat, Lakki Marwat, 28420, KP, Pakistan. ${ }^{4}$ Fixed Point Theory and Applications Research Group, Center of Excellence in Theoretical and Computational Science (TaCS-CoE), Faculty of Science, King Mongkuts University of Technology Thonburi (KMUTT), 126 Pracha Uhit Rd, Bang Mod, Thung Khru, Bagnkok, 10140, Thailand. ${ }^{5}$ Center of Excellence in Theoretical and Computational Science (TaCS-CoE), Faculty of Science, King Mongkuts University of Technology Thonburi (KMUTT), 126 Pracha Uhit Rd, Bang Mod, Thung Khru, Bagnkok, 10140, Thailand. ${ }^{6}$ Department of Medical Research, China Medical University Hospital, China Medical University, Taichung, 40402, Thailand. ${ }^{7}$ NCAO Research Center, Fixed Point Theory and Applications Research Group, Center of Excellence in Theoretical and Computational Science (TaCS-CoE), Faculty of 
Science, King Mongkuts University of Technology Thonburi (KMUTT), 126 Pracha Uhit Rd, Bang Mod, Thung Khru, Bagnkok, 10140, Thailand.

\section{Publisher's Note}

Springer Nature remains neutral with regard to jurisdictional claims in published maps and institutional affiliations.

Received: 16 March 2021 Accepted: 7 June 2021 Published online: 27 July 2021

\section{References}

1. Iftikhar, M., Rehman, H.U., Younis, M.: Solution of thirteenth order boundary value problems by differential transformation method. Asian J. Math. Appl. 2014, Article ID ama0114 (2013)

2. Noor, M.A., Al-Said, E., Mohyud-Din, S.T.: A reliable algorithm for solving tenth-order boundary value problems. Appl. Math. 6(1), 103-107 (2012)

3. Chandrasekhar, S.: Hydrodynamic and Hydromagnetic Stability, vol. 1, 1-354 (2013)

4. Mohyud-Din, S.T., Yildirim, A.: Solutions of tenth and ninth-order boundary value problems by modified variational iteration method. Appl. Appl. Math. 5(1), 11-25 (2010)

5. Siddiqi, S.S., Akram, G., Zulfiqar, l.: Solution of eleventh order boundary value problems using variational iteration technique. Eur. J. Sci. Res. 30(4), 505-525 (2009)

6. Mirmoradi, H., Mazaheripour, H., Ghanbarpour, S., Barari, A.: Homotopy perturbation method for solving twelfth order boundary value problems. Int. J. Res. Rev. Appl. Sci. 1(2), 163-173 (2009)

7. Noor, M.A., Mohyud-Din, S.T.: Solution of twelfth-order boundary value problems by variational iteration technique. J. Appl. Math. Comput. 28(1), 123-131 (2008)

8. Smaher, M.Y.: Efficient modification method of Adomian decomposition method for solving thirteenth order problems. Int. J. Phys. Sci. 7(43), 121-132 (2015)

9. Akram, G., Sadaf, M.: Application of homotopy analysis method to the solution of ninth order boundary value problems in AFTI-F16 fighters. J. Assoc. Arab Univ. Basic Appl. Sci. 24, 149-155 (2017)

10. Akram, G., Nadeem, Z:: Nonpolynomial spline technique for the solution of ninth order boundary value problems. Turk. J. Math. 41(2), 312-325 (2017)

11. Siddiqi, S.S., Twizell, E.H.: Spline solutions of linear tenth-order boundary-value problems. Int. J. Comput. Math. 68(3-4), 345-362 (1998)

12. Siddiqi, S.S., Twizell, E.: Spline solutions of linear twelfth-order boundary-value problems. J. Comput. Appl. Math. 78(2), 371-390 (1997)

13. Lepik, Ü., Hein, H.: Haar wavelets. In: Haar Wavelets, pp. 7-20. Springer, Berlin (2014)

14. Cattani, C.: Haar wavelet splines. J. Interdiscip. Math. 4(1), 35-47 (2001)

15. Chen, C., Hsiao, C.: Haar wavelet method for solving lumped and distributed-parameter systems. IEE Proc., Control Theory Appl. 144(1), 87-94 (1997)

16. Hsiao, C.-H.: State analysis of linear time delayed systems via Haar wavelets. Math. Comput. Simul. 44(5), 457-470 (1997)

17. Lepik, U.: Haar wavelet method for solving higher order differential equations. Int. J. Math. Comput. 1(8), 84-94 (2008)

18. Ali, A., Hussain, I., et al.: Solution of sixth-order boundary-value problems by collocation method using Haar wavelets. Int. J. Phys. Sci. 7(43), 5729-5735 (2012)

19. Ali, A.: Numerical solution of fourth order boundary-value problems using Haar wavelets. Appl. Math. Sci. 5(63), 3131-3146 (2011)

20. Saeed, U., et al.: Assessment of Haar wavelet-quasilinearization technique in heat convection-radiation equations. Appl. Comput. Intell. Soft Comput. 2014, Article ID 454231 (2014)

21. Agarwal, P., Agarwal, R.P., Ruzhansky, M.: Special Functions and Analysis of Differential Equations, vol. 1, pp. 1-371 (2020)

22. Agarwal, P., Ammi, M.S., Asad, J.: Existence and uniqueness results on time scales for fractional nonlocal thermistor problem in the conformable sense. Adv. Differ. Equ. 2021(1), 1 (2021)

23. Chu, Y.-M., Shah, N.A., Agarwal, P., Chung, J.D.: Analysis of fractional multi-dimensional Navier-Stokes equation. Adv. Differ. Equ. 2021(1), 1 (2021)

24. Sunarto, A., Agarwal, P., Sulaiman, J., Chew, J.V.L., Aruchunan, E.: Iterative method for solving one-dimensional fractional mathematical physics model via quarter-sweep and PAOR. Adv. Differ. Equ. 2021(1), 1 (2021)

25. Rajchakit, G., Sriraman, R., Boonsatit, N., Hammachukiattikul, P., Lim, C., Agarwal, P.: Global exponential stability of Clifford-valued neural networks with time-varying delays and impulsive effects. Adv. Differ. Equ. 2021(1), 1 (2021)

26. Ali, M.A., Abbas, M., Budak, H., Agarwal, P., Murtaza, G., Chu, Y.-M.: New quantum boundaries for quantum Simpson's and quantum Newton's type inequalities for preinvex functions. Adv. Differ. Equ. 2021(1), 1 (2021)

27. Rezapour, S., Etemad, S., Tellab, B., Agarwal, P., Garcia Guirao, J.L.: Numerical solutions caused by DGJIM and ADM methods for multi-term fractional BVP involving the generalized $\psi$-RL-operators. Symmetry 13(4), 532 (2021)

28. Bellman, R.E., Quasilinearization, R.K.: Nonlinear Boundary-Value Problems. Elsevier, New York (1965)

29. Majak, J., Shvartsman, B., Kirs, M., Pohlak, M., Herranen, H.: Convergence theorem for the Haar wavelet based discretization method. Compos. Struct. 126, 227-232 (2015)

30. Kasi, K., Raju, Y.S.: Quintic b-spline collocation method for tenth order boundary value problems. Int. J. Comput. Appl. 51(15), 7-13 (2012)

31. Grover, M., Tomer, A.: A new approach to evaluate 12th order boundary value problems with HPM. Int. J. Comput. Math. Numer. Simul. 4(1), 99-112 (2011)

32. Ali, J., Islam, S., Rahim, M.T., Zaman, G.: The solution of special twelfth order boundary value problems by the optimal homotopy asymptotic method. World Appl. Sci. J. 11(3), 371-378 (2010)

33. Adeosun, T.A., Fenuga, O.J., Adelana, S.O., John, A.M., Olalekan, O., Alao, K.B.: Variational iteration method solutions for certain thirteenth order ordinary differential equations (2013) 